\title{
ESSENTIAL OILS FROM LEAVES OF Cryptocarya spp FROM THE ATLANTIC RAIN FOREST
}

\author{
Marcelo Telascrea, Carla C. de Araújo and Alberto J. Cavalheiro* \\ Departamento de Química Orgânica, Instituto de Química, Universidade Estadual de São Paulo, CP 355, 14801-970 Araraquara
} - SP, Brazil

Márcia O. M. Marques and Roselaine Facanali

Centro de Genética, Biologia Molecular e Fitoquímica, Instituto Agronômico de Campinas, 13020-902 Campinas - SP, Brazil Pedro L. R. de Moraes

Departamento de Botânica, Instituto de Biologia, Universidade Estadual de Campinas, 13083-970 Campinas - SP, Brazil

Recebido em 11/12/06; aceito em 6/9/07; publicado na web em 10/3/08

\begin{abstract}
The essential oils from leaves of four Cryptocarya spp endemic in the Brazilian Atlantic rain forest were obtained by hydrodistillation and shown by GC-MS analysis to contain mono and sesquiterpenes. The major components of the oil of Cryptocarya moschata were linalool (34.3\%), $\alpha$-terpinene (17.0\%), $\gamma$-terpinene (10.4\%), 1,8-cineole (5.8\%) and trans-ocimene (4.8\%), whilst those of C. botelhensis were $\alpha$-pinene (22.7\%), $\beta$-pinene $(9.2 \%)$, trans-verbenol $(8.4 \%)$, trans-pinocarveol $(5.5 \%)$ and myrtenal $(5.4 \%)$. The principal compounds of $C$. mandioccana oil were $\beta$-caryophyllene (13.8\%), spathulenol (10.2\%), caryophyllene oxide (7.8\%), $\delta$-cadinene (6.9\%) and bicyclogermacrene (6.4\%), whilst those of C. saligna were germacrene D (15.5\%), bicyclogermacrene (13.8\%), spathulenol (11.8\%) and germacrene B (5.7\%).
\end{abstract}

Keywords : essential oils; Cryptocarya spp.; Lauraceae.

\section{INTRODUCTION}

Cryptocarya (Lauraceae) is a pantropical genus containing around 350 species distributed mainly within Malaysia and Australia. ${ }^{1}$ Although 23 species have been described for South America, de Moraes $^{2}$ has recognized eight validly published species names. Seven of these species, namely, Cryptocarya aschersoniana Mez, C. citriformis (Vell.) P.L.R. de Moraes, C. mandioccana Meisn., C. micrantha Meisn., C. moschata Nees \& Mart., C. saligna Mez and C. subcorymbosa Mez. are endemic in Atlantic Rain Forest (Mata Atlântica) of Brazil.

In earlier phytochemical investigations of the essential oils of Cryptocarya species, the occurrence of (-)-massoilactone was reported in the bark oil of $C$. massoia ${ }^{3}$ and linalool was reported in leaf oil of $C$. moschata and $C$. aschersoniana, although the last of these also contained $\beta$-myrcene, 1,8-cineol, and stereo-isomeric linalool oxides. ${ }^{4}$ Seventy one components were identified in the essential oil from leaves of $C$. $a l b a$, the most important of which were terpin-4-ol, $p$-cymol, cineol, $\alpha$-pinene, $\beta$-pinene and borneol-terpineol. ${ }^{5}$ The essential oil from leaves of C. cunninghamii contains either bicyclogermacrene $(52.4 \%)$ or 6nonyl-5,6-dihydro-5,6-pyrone (78-88\%) as its major component, besides 6-heptyl-5,6-dihydro-2H-pyran-2-one, 6-pentyl-5,6-2H-pyran-2-one and 2-phenylethyl benzoate. ${ }^{6}$ Analysis of essential oils obtained by hydrodistillation of leaves of $C$. mandioccana, including intraspecific variants, yielded 64 compounds with predominance of $\beta$-caryophyllene, spathulenol, caryophyllene oxide, $\delta$-cadinene, germacrene D, benzaldehyde and bicyclogermacrene. ${ }^{7}$ Sampling the essential oil from leaves of $C$. moschata Nees (current status C. mandioccana Meisner) at different times and seasons revealed no significant variation in oil composition. $^{8}$

In the present study, we have compared the chemical constitutions of the essential oil from leaves of $C$. mandioccana, $C$. moschata and $C$. saligna, the three most common Cryptocarya

*e-mail: albjcava@iq.unesp.br species from Atlantic Rain Forest of São Paulo State, Brazil, and C. botelhensis - a new species described in 2006 by de Moraes. ${ }^{9}$

\section{EXPERIMENTAL}

\section{Plant material}

Leaves of C. mandioccana Meisn. and C. botelhensis P. L. R. Moraes were harvested in November 1999 and December 2000, respectively, from the Parque Estadual Carlos Botelho, São Miguel Arcanjo, São Paulo. Leaves of C. moschata Nees \& Mart. were collected in February 2002 from the Mata da Mariana, Ibaté, São Paulo, whilst leaves of $C$. saligna Mez were gathered in November 1999 from the Parque Estadual Serra do Mar - Núcleo Picinguaba, Ubatuba, São Paulo. All the species were in fruits at the date of collection except $C$. botelhensis, which was in bud. Plant material was identified by Dr. P. L. R. de Moraes. A voucher specimen of $C$. mandioccana (Cavalheiro CB353) was deposited in the São Paulo State herbarium "Maria Eneyda P. Kaufmann Fidalgo" (SP) and vouchers of $C$. botelhensis, $C$. saligna and $C$. moschata (Moraes 1243, Moraes 2472, and Moraes 2347, respectively)were deposited in Escola Superior de Agricultura Luís de Queiroz herbarium (ESA).

\section{Hydrodistillation}

Fresh leaf material (around $500 \mathrm{~g}$ ) was frozen with liquid nitrogen, triturated using a porcelain mortar and pestle and submitted to hydrodistillation (i. e. plant material in boiling water) in a Clevengertype apparatus to yield 0.05 to $0.06 \%$ of oil. The oil was stored at -18 ${ }^{\circ} \mathrm{C}$ in a glass vial under nitrogen until required for further analysis.

\section{Essential oil analysis}

Analyses were performed using a Shimadzu model QP-5000 gas chromatograph-mass spectrometer equipped with a DB-5 (J\&W 
Scientific) fused silica capillary column $(30 \mathrm{~m} \times 0.25 \mathrm{~mm} ; 0.25$ $\mu \mathrm{m})$. The oven temperature was programmed from 60 to $240{ }^{\circ} \mathrm{C}$ at 3 ${ }^{\circ} \mathrm{C} / \mathrm{min}$, and the injector and interface temperatures were maintained at 240 and $230{ }^{\circ} \mathrm{C}$, respectively. ${ }^{10}$ The carrier gas was helium at a flow rate of $1.0 \mathrm{~mL} \mathrm{~min}{ }^{-1}$ (constant volume). Mass spectra were obtained at $70 \mathrm{eV}$. Samples $(1 \mu \mathrm{L})$ were injected manually in the split mode (20:1). Identifications of essential oil components were made by reference to the NIST 62 library, ${ }^{11}$ and their relative percentages were calculated from normalized peak areas (uncorrected for specific responses). Retention indices ${ }^{12}$ were determined relative to the retention times of a series of $n$-alkane standards (C-10 to C-30: Sigma, product ref. no. R 8769, Sigma), measured under the same chromatographic conditions described above, and compared with published values. ${ }^{10}$

\section{RESULTS AND DISCUSSION}

Ninety compounds present in the essential oils from leaves of four Cryptocarya species were identified from chromatographic and spectrometric data (Table 1). The chemical profiles of these oils showed significant differences in composition between the species studied (Figure 1). The main constituents of C. mandioccana oil were sesquiterpenes ( $\beta$-caryophyllene, spathulenol, caryophyllene oxide, $\delta$-cadinene, bicyclogermacrene and germacrene D), whilst those of the oil of $C$. moschata were acyclic and menthane type monoterpenes (linalool and $\alpha$-terpinene) as previously reported for these species. ${ }^{4,7}$ The main constituents of the essential oil of $C$. botelhensis were the pinane monoterpenes, whilst sesquiterpenes were predominant in the essential oil of C. saligna.

The major sesquiterpenes identified in the essential oil from leaves of C. mandioccana belongs to two main classes (Figure 2), namely, the humulene/caryophyllene type resulting from $\mathrm{C} 1-\mathrm{C} 11$ cyclisation of farnesyl diphosphate (FPP), and those derived from germacrene/ bicyclogermacrene produced by $\mathrm{C} 1-\mathrm{C} 10$ cyclisation of the $\mathrm{C}_{15}$-precursor. ${ }^{7,13,14}$ The occurrence of distinct sesquiterpene synthases, each associated with a different mode of cyclisation of FPP, is not yet conclusive and it has been suggested that a certain degree of freedom in the process of ring closure might be responsible for the formation of multiple end-products. ${ }^{15}$ However, the essential oil from C. saligna exhibited a significant predominance of sesquiterpenes deriving from C1-C10 cyclisation and this may be indicative of the occurrence of a specific sesquiterpene synthase within this species.

Table 1. Constituents of the essential oil from leaves of Cryptocarya spp. endemic in the Brazilian Atlantic rain forest

\begin{tabular}{|c|c|c|c|c|c|c|c|c|c|c|}
\hline \multirow[b]{2}{*}{ No. } & \multirow[b]{2}{*}{ Compound } & \multirow[b]{2}{*}{$\mathrm{RI}_{\text {lit }}$} & \multicolumn{2}{|c|}{ C. mandioccana } & \multicolumn{2}{|c|}{ C. moschata } & \multicolumn{2}{|c|}{ C. saligna } & \multicolumn{2}{|c|}{ C. botelhensis } \\
\hline & & & $\mathrm{RI}_{\text {exp }}$ & $\%$ & $\mathrm{RI}_{\text {exp }}$ & $\%$ & $\mathrm{RI}_{\text {exp }}$ & $\%$ & $\mathrm{RI}_{\text {exp }}$ & $\%$ \\
\hline 1 & $\alpha$-thujene & 931 & & & 925 & 1.4 & 928 & 0.2 & & \\
\hline 2 & $\alpha$-pinene & 939 & 939 & 1.7 & 932 & 2.3 & 933 & 4.3 & 935 & 22.7 \\
\hline 3 & $\alpha$-fenchene & 951 & & & & & & & 947 & 0.6 \\
\hline 4 & camphene & 953 & 947 & 0.2 & 955 & 0.4 & & & & \\
\hline 5 & ni & - & & & & & 947 & 6.9 & & \\
\hline 6 & thuja-2.4(10)-diene & 957 & & & & & & & 952 & 1.2 \\
\hline 7 & ni & - & & & & & 955 & 0.4 & & \\
\hline 8 & benzaldehyde & 961 & 957 & 3.4 & & & & & & \\
\hline 9 & sabinene & 976 & & & 972 & 2.6 & 972 & 5.6 & & \\
\hline 10 & $\beta$-pinene & 980 & 976 & 1.0 & 976 & 3.1 & 976 & 2.6 & 977 & 9.2 \\
\hline 11 & $\beta$-mircene & 991 & & & 990 & 1.9 & & & & \\
\hline 12 & $\alpha$-terpinene & 1018 & & & 1016 & 17.0 & & & 1016 & 0.3 \\
\hline 13 & $o$-cymene & 1022 & & & 1022 & 3.1 & 1023 & 0.3 & 1023 & 0.4 \\
\hline 14 & $p$-menthene & 1023 & & & & & & & 1025 & 0.3 \\
\hline 15 & $\beta$-felandrene & 1031 & & & 1026 & 1.6 & & & & \\
\hline 16 & limonene & 1031 & & & & & 1027 & 0.6 & 1027 & 0.2 \\
\hline 17 & benzyl alcohol & 1032 & & & & & 1029 & 1.4 & & \\
\hline 18 & 1.8-cineol & 1033 & & & 1028 & 5.8 & & & 1030 & 3.4 \\
\hline 19 & cis-ocimene & 1040 & & & 1035 & 0.5 & & & & \\
\hline 20 & trans-ocimene & 1050 & & & 1045 & 4.8 & & & & \\
\hline 21 & $\gamma$-terpinene & 1062 & & & 1056 & 10.4 & 1057 & 0.2 & 1057 & 0.3 \\
\hline 22 & acetophenone & 1065 & 1069 & 0.5 & & & & & & \\
\hline 23 & cis-sabinene hydrate & 1068 & & & & & 1065 & 0.2 & & \\
\hline 24 & cis-linalool oxide & 1074 & 1074 & 1.0 & 1067 & 0.7 & & & & \\
\hline 25 & allyl hexanoate & 1080 & & & & & 1069 & 0.1 & & \\
\hline 26 & trans-linalool oxide & 1088 & 1084 & 0.8 & & & & & & \\
\hline 27 & terpinolene & 1088 & & & 1082 & 0.8 & & & 1082 & 0.6 \\
\hline 28 & $\mathrm{ni}$ & - & & & & & & & 1086 & 0.3 \\
\hline 29 & trans-sabinene hydrate & 1097 & & & & & 1097 & 0.2 & & \\
\hline 30 & 6-camphenone & 1093 & & & & & & & 1088 & 0.6 \\
\hline 31 & linalool & 1098 & 1097 & 0.7 & 1096 & 34.3 & 1091 & 0.8 & 1094 & 1.4 \\
\hline 32 & ni & 1098 & 1102 & 0.5 & & & & & & \\
\hline 33 & isopenthyl 2-methyl butanoate & 1099 & & & & & & & 1103 & 0.2 \\
\hline 34 & isopentyl isovalerate & 1103 & & & & & 1102 & 0.5 & & \\
\hline 35 & trans-pinan-2-ol & 1119 & & & & & & & 1120 & 0.1 \\
\hline 36 & $\alpha$-campholenal & 1125 & & & & & 1123 & 0.5 & 1125 & 2.6 \\
\hline
\end{tabular}


Table 1. continuation

\begin{tabular}{|c|c|c|c|c|c|c|c|c|c|c|}
\hline \multirow[b]{2}{*}{ No. } & \multirow[b]{2}{*}{ Compound } & \multirow[b]{2}{*}{$\mathrm{RI}_{\text {lit }}$} & \multicolumn{2}{|c|}{ C. mandioccana } & \multicolumn{2}{|c|}{ C. moschata } & \multicolumn{2}{|c|}{ C. saligna } & \multicolumn{2}{|c|}{ C. botelhensis } \\
\hline & & & $\mathrm{RI}_{\text {exp }}$ & $\%$ & $\mathrm{RI}_{\text {exp }}$ & $\%$ & $\mathrm{RI}_{\text {exp }}$ & $\%$ & $\mathrm{RI}_{\text {exp }}$ & $\%$ \\
\hline 37 & cis-limonene oxide & 1134 & & & & & 1029 & 0.1 & & \\
\hline 38 & nopinone & 1137 & & & & & & & 1134 & 0.4 \\
\hline 39 & trans-pinocarveol & 1139 & & & & & 1137 & 0.7 & 1138 & 5.5 \\
\hline 40 & cis-verbenol & 1140 & & & & & & & 1140 & 1.0 \\
\hline 41 & trans-verbenol & 1144 & & & & & 1142 & 1.3 & 1146 & 8.4 \\
\hline 42 & cis-dehidro- $\alpha$-terpineol & 1144 & & & & & & & 1148 & 0.2 \\
\hline 43 & sabina ketone & 1156 & & & & & 1154 & 0.3 & & \\
\hline 44 & trans-pinocamphone & 1160 & & & & & & & 1158 & 0.3 \\
\hline 45 & pinocarvone & 1162 & & & & & 1159 & 0.3 & 1161 & 2.5 \\
\hline 46 & $p$-mentha-1.5-dien-8-ol & 1166 & & & & & & & 1164 & 0.7 \\
\hline 47 & terpin-4-ol & 1177 & & & 1179 & 0.9 & 1175 & 0.7 & & \\
\hline 48 & naphthalene & 1179 & & & & & 1183 & 0.3 & & \\
\hline 49 & $p$-cymen-2-ol & 1183 & & & & & & & 1183 & 0.3 \\
\hline 50 & $\alpha$-terpineol & 1189 & & & & & & & 1184 & 0.1 \\
\hline 51 & myrtenal & 1193 & & & & & 1194 & 0.8 & 1194 & 5.4 \\
\hline 52 & verbenone & 1204 & & & & & 1207 & & 1207 & 2.4 \\
\hline 53 & trans-carveol & 1217 & & & & & & & 1217 & 0.7 \\
\hline 54 & carvone & 1242 & & & & & & & 1240 & 0.1 \\
\hline 55 & bornyl acetate & 1285 & & & & & 1285 & 0.6 & & \\
\hline 56 & $\delta$-elemene & 1339 & 1337 & 0.3 & & & 1337 & 1.8 & & \\
\hline 57 & $\alpha$-cubebene & 1351 & 1350 & 0.8 & & & & & 1349 & 0.4 \\
\hline 58 & $\alpha$-copaene & 1376 & 1376 & 2.9 & & & 1375 & 1.6 & 1376 & 1.2 \\
\hline 59 & $\beta$-bourbonene & 1384 & 1384 & 0.8 & & & 1384 & 0.5 & & \\
\hline 60 & $\beta$-cubebene & 1390 & 1390 & 0.5 & & & 1391 & 0.5 & 1389 & 0.2 \\
\hline 61 & $\beta$-elemene & 1391 & 1392 & 2.6 & & & 1393 & 2.8 & & \\
\hline 62 & ni & - & & & & & & & 1395 & 0.4 \\
\hline 63 & $\beta$-caryophyllene & 1418 & 1418 & 13.8 & 1418 & 3.3 & 1419 & 4.7 & 1419 & 0.6 \\
\hline 64 & $\beta$-cedrene & 1418 & 1420 & 0.3 & & & & & & \\
\hline 65 & $\beta$-gurjunene & 1432 & 1430 & 0.4 & & & & & & \\
\hline 66 & aromadendrene & 1439 & 1438 & 0.4 & & & & & & \\
\hline 67 & $\gamma$-elemene & 1433 & & & & & 1433 & 0.8 & & \\
\hline 68 & $\alpha$-humulene & 1454 & 1453 & 2.1 & 1460 & 0.5 & 1453 & 1.3 & & \\
\hline 69 & allo-aromadendrene & 1461 & 1461 & 2.6 & & & 1460 & 0.3 & 1461 & 3.1 \\
\hline 70 & $\gamma$-muurolene & 1477 & 1476 & 1.4 & & & & & 1477 & 0.5 \\
\hline 71 & germacrene D & 1480 & 1481 & 4.5 & & & 1481 & 15.5 & & \\
\hline 72 & ar-curcumene & 1483 & & & & & & & 1482 & 1.2 \\
\hline 73 & $\beta$-selinene & 1485 & 1486 & 1.4 & & & & & & \\
\hline 74 & phenyl ethyl 3-methylbutanoate & 1489 & & & & & & & 1486 & 2.7 \\
\hline 75 & valencene & 1491 & 1491 & 0.8 & & & & & & \\
\hline 76 & epi-cubebol & 1493 & & & & & & & 1495 & 2.5 \\
\hline 77 & bicyclogermacrene & 1494 & 1496 & 6.4 & & & 1497 & 13.8 & & \\
\hline 78 & $\alpha$-muurolene & 1499 & 1502 & 1.2 & & & & & & \\
\hline 79 & $\gamma$-cadinene & 1513 & 1514 & 1.3 & & & & & 1516 & 2.3 \\
\hline 80 & $\dot{\delta}$-cadinene & 1524 & 1528 & 6.9 & & & 1523 & 2.2 & & \\
\hline 81 & cadina-1.4-diene & 1532 & 1531 & 0.3 & & & & & & \\
\hline 82 & $\alpha$-calacorene & 1542 & 1542 & 0.4 & & & & & & \\
\hline 83 & germacrene B & 1556 & & & 1566 & 1.0 & 1556 & 5.7 & & \\
\hline 84 & spathulenol & 1576 & 1578 & 10.2 & & & 1577 & 11.8 & 1582 & 6.1 \\
\hline 85 & trans-sesquisabinene hydrate & 1580 & & & & & & & 1586 & 4.9 \\
\hline 86 & carophyllene oxide & 1581 & 1583 & 7.8 & & & 1582 & 1.9 & & \\
\hline 87 & globulol & 1583 & & & & & 1589 & 0.6 & & \\
\hline 88 & viridiflorol & 1590 & 1589 & 2.8 & & & & & & \\
\hline 89 & guaiol & 1595 & 1595 & 1.0 & & & & & & \\
\hline 90 & $\mathrm{ni}$ & - & 1604 & 2.6 & & & & & & \\
\hline 91 & ni & - & 1609 & 0.8 & & & & & & \\
\hline 92 & epi-cubenol & 1627 & 1628 & 1.9 & & & & & & \\
\hline 93 & ni & - & 1638 & 0.9 & & & & & & \\
\hline 94 & cubenol & 1642 & 1642 & 3.1 & & & & & & \\
\hline 95 & $\alpha$-muurolol & 1645 & 1645 & 0.8 & & & & & & \\
\hline 96 & ni & - & 1649 & 1.1 & & & & & & \\
\hline 97 & selin-11-en- $\alpha$-ol $\alpha$-cadinol & 1653 & 1653 & 3.2 & & & & & & \\
\hline 98 & 14-hydroxy-9-epi-caryophyllene & e 1664 & 1657 & 0.7 & & & & & & \\
\hline 99 & ni & - & 1673 & 0.8 & & & & & & \\
\hline 100 & khusinol & 1674 & 1687 & 0.5 & & & & & & \\
\hline
\end{tabular}

$\mathrm{RI}_{\text {lit }}=$ retention index from literature; $\mathrm{RI}_{\text {exp }}=$ experimental retention index; \% = normalized percentage; ni = not identified 

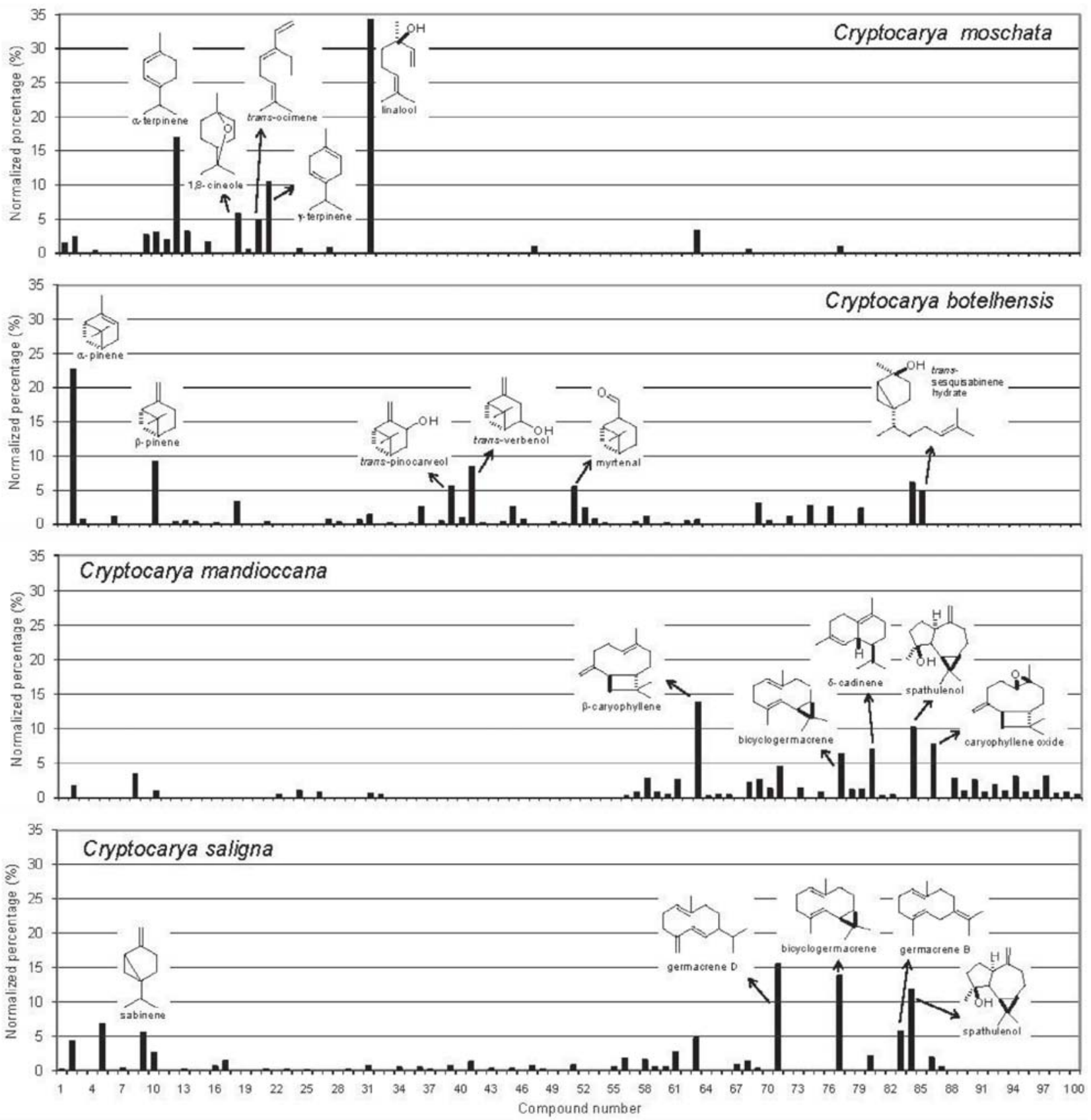

Figure 1. Chemical profile of essential oils from leaves of Cryptocarya spp. For the key to peak identity, see Table 1

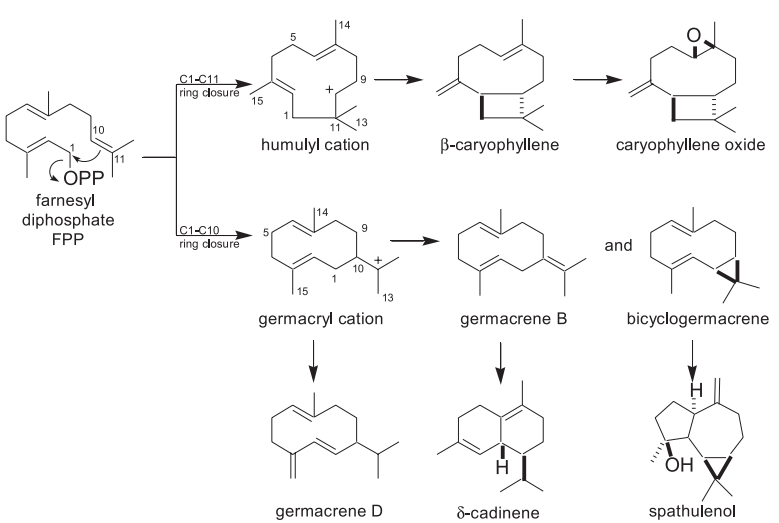

Figure 2. Biogenetic relationships between the main sesquiterpenes of Cryptocarya mandioccana and $\mathrm{C}$. saligna

\section{CONCLUSION}

The chemical profile of essential oils from leaves of four members of the genus Cryptocarya was characteristic to each of the species studied. The oil of $C$. moschata presented a predominance of acyclic (linalool, trans-ocimene) and menthane $(\alpha$-terpinene, $\gamma$-terpinene, 1,8-cineole) monoterpenes, whilst pinane monoterpenes ( $\alpha$-pinene, $\beta$-pinene, trans-verbenol, transpinocarveol, myrtenal) were the main components in the oil of $C$. botelhensis. Sesquiterpenes derived from C1-C11 ( $\beta$-caryophyllene, caryophyllene oxide) and $\mathrm{C} 1-\mathrm{C} 10$ (spathulenol, $\delta$-cadinene, bicyclogermacrene) cyclization of FPP were the main constituents of $C$. mandioccana oil, although the principal components of the oil of $C$. saligna were sesquiterpenes derived from C1-C10 cyclisation of the FPP precursor (germacrene D, bicyclogermacrene, spathulenol, germacrene B). 


\section{ACKNOWLEDGMENTS}

The authors are grateful to FAPESP for financial support and to CAPES and CNPq for scholarships to M. Telascrea and C. C. de Araújo, respectively. We acknowledge Instituto Florestal for authorizing the collection of Cryptocarya spp at Parque Estadual Carlos Botelho and Parque Estadual da Serra do Mar.

\section{REFERENCES}

1. Rohwer, J. G. In The families and genera of Vascular Plants, II - Flowering Plants, Dicotyledons; Kubitzki, K.; Rohwer, J.G.; Bittrich, V., eds.; SpringerVerlag: Berlin, 1993, p. 366

2. de Moraes, P. L. R.; Taxon 2005, 54, 789

3. Abe, S. J.; Chem. Soc. Japan 1937, 58, 246.

4. Naves, Y. R. T.; Alves H. M.; Arrndt, V. H.; Gottlieb, O. R.; Magalhães, M. T.; Helv. Chim. Acta 1975, 46, 102.
5. Montes, M.; Valenzuela, L.; Wilkomirsky, T.; Sanguinetti, A.; von Bauer, D.; Ann. Pharm. Fr. 1988, 46, 41.

6. Brophy, J. J.; Goldsack, R. J.; Forster, P. I.; J. Essent. Oil Res. 2001, 13, 332.

7. Telascrea, T.; Araújo, C. C.; Marques, M. O. M.; Facanali, R.; Moraes, P. L. R.; Cavalheiro, A. J.; Biochem. Syst. Ecol. 2007, 35, 222.

8. Marchetti, C. N.; Telascrea, M.; Tininis, A. G.; Cavalheiro, A. J.; Rev. Bras. Plantas Med. 2006, 8, 23.

9. de Moraes, P. L. R.; Taxonomy of Cryptocarya species of Brazil, ABC Taxa, Series, Royal Belgian Institute of Natural Sciences: Brussels, 2007, in press.

10. Adams, R. P.; Identification of Essential Oil by Ion Trap Mass Spectroscopy, Academic Press: San Diego, 1995.

11. McLafferty, F. W.; Stauffer, D.; The Wiley/NBS Registry of Mass Spectral Data, John Wiley Sons: New York, 1989

12. van Den Dool, H.; Kratz, P. D.; J. Chromatogr. 1963, 11, 463

13. Yoshihara, K.; Ohta, Y.; Sakai, T.; Hirose, Y.; Tetrahedron Lett. 1969, 2263.

14. Bülow, N.; König, W. A.; Phytochemistry 2000, 55, 141.

15. van der Hoeven, R. S.; Monforte, A. J.; Breeden, D.; Tanksley, S. D.; Steffens, J. C.; Plant Cell. 2000, 12, 2283. 